\title{
First-Order Side-Channel Attacks on the Permutation Tables Countermeasure
}

\author{
Emmanuel Prouff ${ }^{1}$ and Robert $\mathrm{McEvoy}^{2}$ \\ 1 Oberthur Technologies, France \\ e.prouff@oberthur.com \\ 2 Claude Shannon Institute for Discrete Mathematics, Coding and Cryptography, \\ University College Cork, Ireland \\ robertmce@eleceng.ucc.ie
}

\begin{abstract}
The use of random permutation tables as a side-channel attack countermeasure was recently proposed by Coron [5]. The countermeasure operates by ensuring that during the execution of an algorithm, each intermediate variable that is handled is in a permuted form described by the random permutation tables. In this paper, we examine the application of this countermeasure to the AES algorithm as described in [5], and show that certain operations admit first-order side-channel leakage. New side-channel attacks are developed to exploit these flaws, using correlation-based and mutual information-based methods. The attacks have been verified in simulation, and in practice on a smart card.
\end{abstract}

Keywords: Side-Channel Attacks, Permutation Tables, CPA, MIA, Masking.

\section{Introduction}

When a cryptographic algorithm is implemented in hardware or embedded software, information may be leaked about the intermediate variables being processed by the device. The class of implementation attacks called Side-Channel Attacks (SCA) aims to exploit these leakages, and recover secret information [11. Masking is one of the most popular SCA countermeasures, used to protect sensitive variables (i.e. variables whose statistical distribution is dependent on the secret key) [4]. Masking has been well studied, and has been shown to be effective against a number of types SCA [24], but remains ineffective in stronger attack models (e.g. Higher-Order SCA [13]).

Recently, Coron presented the permutation tables countermeasure, as an alternative to masking [5]. The new proposal can be viewed as a generalization of the classical approach, where masking is no longer performed through a random translation, but through a random permutation. Like classical masking, the permutation tables countermeasure also requires a random bit string, which is used at the start of the cryptographic algorithm to generate a permutation $P$. In the case of an encryption algorithm, $P$ is then applied to both the message $x$ to be encrypted and the secret key $k$, producing $P(x)$ and $P(k)$ respectively. It 
is these permuted variables that are used by the encryption algorithm. At each stage of the algorithm, the cryptographic operations must be modified so that all of the intermediate variables remain in the permuted form described by $P$. If the countermeasure is applied correctly, the intermediate variables should all have a uniform distribution independent of sensitive variables, thereby precluding side-channel attacks that rely on statistical dependency of the intermediate variables with the secret key.

In this paper, we examine the application of the permutation tables countermeasure to AES, as described by 5 . We show that certain sensitive intermediate variables in this algorithm are, in fact, not uniformly distributed, and therefore leak side-channel information about the secret key. However, because of the nature of the permutation tables countermeasure, it is not possible to exploit these flaws with classical approaches (such as those used in 6 7/9]). In fact, the main issue is to exhibit a sound prediction function to correlate with the leakages in correlation-based SCA (e.g. Correlation Power Analysis (CPA) [3]). After modeling the side-channel leakage, we use the method proposed in [17] to exhibit a new prediction function for the permuted sensitive variables. An analytical expression for the optimal prediction function is derived, which, for the correct key hypothesis, maximises the correlation with leakage measurements from the algorithm.

Furthermore, since the flawed intermediate variables do not have a monotonic dependency with the sensitive variables, we consider SCA attacks involving distinguishers able to exploit non-monotonic interdependencies. We investigate how Mutual Information Analysis (MIA) 816] can be applied in order to exploit the flaws, and compare it with the correlation-based approach. Both of these new attacks are performed both in simulation and in practice on a smart card, and are successful at breaking the countermeasure described in [5].

\section{Preliminaries}

\subsection{Mathematical Background and Notation}

We use calligraphic letters, like $\mathcal{X}$, to denote finite sets $\left(e . g . \mathbb{F}_{2}^{n}\right)$. The corresponding capital letter $X$ is used to denote a random variable over $\mathcal{X}$, while the lowercase letter $x$ denotes a particular element from $\mathcal{X}$. The probability of the event $(X=x)$ is denoted $\mathrm{p}[X=x]$. The uniform probability distribution over a set $\mathcal{X}$ is denoted by $\mathcal{U}(\mathcal{X})$, and the Gaussian probability distribution with mean $\mu$ and standard deviation $\sigma$ is denoted by $\mathcal{N}\left(\mu, \sigma^{2}\right)$. The mean of $X$ is denoted by $\mathrm{E}[X]$ and its standard deviation by $\sigma[X]$. The correlation coefficient between $X$ and $Y$ is denoted by $\rho[X, Y]$. It measures the linear interdependence between $X$ and $Y$, and is defined by:

$$
\rho[X, Y]=\frac{\operatorname{Cov}[X, Y]}{\sigma[X] \sigma[Y]},
$$


where $\operatorname{Cov}[X, Y]$, called covariance of $X$ and $Y$, equals $\mathrm{E}[X Y]-\mathrm{E}[X] \mathrm{E}[Y]$. It can be checked [17] that for every function $f$ measurable on $\mathcal{X}$, the correlation $\rho[f(X), Y]$ satisfies:

$$
\rho[f(X), Y]=\rho[f(X), \mathrm{E}[Y \mid X]] \times \rho[\mathrm{E}[Y \mid X], Y] .
$$

This implies (see Proposition 5 in [17]) the following inequality:

$$
\rho[f(X), Y] \leq \frac{\sigma[\mathrm{E}[Y \mid X]]}{\sigma[Y]} .
$$

A sample of a finite number of values taken by $X$ over $\mathcal{X}$ is denoted by $\left(x_{i}\right)_{i}$ or by $\left(x_{i}\right)$ if there is no ambiguity on the index, and the mean of such a sample is denoted by $\bar{x}=\frac{1}{\#\left(x_{i}\right)} \sum_{i} x_{i}$. Given two sample sets $\left(x_{i}\right)$ and $\left(y_{i}\right)$, the empirical version of the correlation coefficient is the Pearson coefficient:

$$
\widehat{\rho}\left(\left(x_{i}\right),\left(y_{i}\right)\right)=\frac{\sum_{i}\left(x_{i}-\bar{x}\right)\left(y_{i}-\bar{y}\right)}{\sqrt{\sum_{i}\left(x_{i}-\bar{x}\right)^{2}} \sqrt{\sum_{i}\left(y_{i}-\bar{y}\right)^{2}}},
$$

The correlation and Pearson coefficients relate to affine statistical dependencies, and two dependent variables $X$ and $Y$ can be such that $\rho(X, Y)=0$. To quantify the amount of information that $Y$ reveals about $X$ (whatever the kind of dependency is), the notion of mutual information is usually involved. It is the value $\mathrm{I}(X ; Y)$ defined by $\mathrm{I}(X ; Y)=\mathrm{H}(X)-\mathrm{H}(X \mid Y)$, where $\mathrm{H}(X)$ is the entropy of $X$ and where $\mathrm{H}(X \mid Y)$ is the conditional entropy of $X$ knowing $Y$ (see 12 for more details).

\subsection{Side-Channel Attack Terminology}

We shall view an implementation of a cryptographic algorithm as the processing of a sequence of intermediate variables, as defined in 2. We shall say that an intermediate variable is sensitive if its distribution is a function of some known data (for example, the plaintext) and the secret key, and is not constant with respect to the secret key. Consequently, the statistical distribution of a sensitive variable depends on both the key and on the distribution of the known data. If a sensitive intermediate variable appears during the execution of a cryptographic algorithm, then that implementation is said to contain a first-order flaw. Information arising from a first-order flaw, that can be monitored via a side-channel (such as timing information or power consumption), is termed first-order leakage. A first-order side-channel attack (SCA) against an implementation is a SCA that exploits a first-order leakage, in order to recover information about the secret key. Similarly, an rth-order SCA (Higher-Order $S C A$ or $H O-S C A$ ) against an implementation is a SCA that exploits leakages at $r$ different times, which are respectively associated with $r$ different intermediate variables.

Remark 1. In [19], an alternative definition for HO-SCA is used, where an $r$ thorder SCA is defined with respect to $r$ different algorithmic variables (which may 
be manipulated simultaneously, or which may correspond to a single intermediate variable). In this paper, we focus on the countermeasure of [5]; therefore, we adhere to the HO-SCA definition in [5] (which is widely accepted in the community [2101314]).

In order to prevent side-channel attacks on cryptographic implementations, many countermeasures (such as masking and the permutation tables countermeasure) aim to randomise the leakage caused by each intermediate variable. An implementation of a cryptographic algorithm can be said to possess first-order $S C A$ security if no intermediate variable in the implementation is sensitive. Similarly, $r$ th-order SCA security requires an implementation to be such that no $r$-tuple of its intermediate variables is sensitive.

\section{The Permutation Tables Countermeasure}

\subsection{Generation of Permutation Tables}

In order to use permutation tables as a SCA countermeasure, a new permutation table $P$ must be generated at the beginning of each execution of the cryptographic algorithm. Here, $P$ is described in the context of the AES algorithm, where the intermediate variables are 8 -bit words. $P$ comprises two 4 -bit permutations $p_{1}$ and $p_{2}$, and operates on an 8 -bit variable $x$ according to:

$$
P(x)=p_{2}\left(x_{h}\right)|| p_{1}\left(x_{l}\right),
$$

where $x_{h}$ and $x_{l}$ respectively denote the high and low nibbles of $x$, and $\|$ denotes concatenation. Upon each invocation of the algorithm, permutations $p_{1}$ and $p_{2}$ are randomly chosen from a set of permutations $\mathcal{P}$, defined over $\mathbb{F}_{2}^{4}$. For efficiency reasons, the set $\mathcal{P}$ is not defined as the set of all the permutations over $\mathbb{F}_{2}^{4}$. Indeed, in such a case the random generation of an element of $\mathcal{P}$ would be costly. In [5], Coron defines an algorithm to generate elements of the set $\mathcal{P}$ from a 16-bit random value. Here, we will assume that the random variable $P_{1}$ (respectively $P_{2}$ ) associated with the random generation of $p_{1}$ (resp. $p_{2}$ ) satisfies $\mathrm{p}\left[P_{1}=p_{1}\right]=1 / \# \mathcal{P}\left(\right.$ resp. $\left.\mathrm{p}\left[P_{2}=p_{2}\right]=1 / \# \mathcal{P}\right)$ for every $p_{1} \in \mathcal{P}\left(\operatorname{resp} . p_{2} \in \mathcal{P}\right)$.

\subsection{Protecting AES Using Permutation Tables}

The Advanced Encryption Standard (AES) is a well-known block cipher, and details of the algorithm can be found in [5]. Essentially, the AES round function for encryption operates on a 16-byte state (with each element labelled $a_{i}, 0 \leq i \leq$ 15), and consists of four transformations: AddRoundKey, SubBytes, ShiftRows and MixColumns.

In [5], Coron described how to protect the AES encryption algorithm against side-channel attacks, by using the permutation tables countermeasure. We will refer to this encryption algorithm as randomised AES. Firstly, after the random permutation $P$ has been generated (as described in [5]), it is applied to each byte 
of both the message and the key. For every byte $x$, we will refer to $u=P(x)$ as the $P$-representation of $x$. Each permuted value is passed to the AES round function, where it is operated upon by the AES transformations listed above. As noticed by Coron in [5], each of these AES transformations must be carefully implemented, such that: (i) sensitive variables always appear in their $P$-representation, and (ii) the output of each transformation is in $P$-representation form. Coron described the following implementations of AddRoundKey and MixColumns (for details of the other transformations, see [5]):

- Randomised AddRoundKey takes two bytes $u=P(x)$ and $v=P(y)$ as inputs, and outputs $P(x \oplus y)$. In order to achieve this, two 8-bit to 4-bit tables are defined (for $\left(u_{l}, v_{l}\right)-$ resp. $\left(u_{h}, v_{h}\right)-$ in $\left.\left(\mathbb{F}_{2}^{4}\right)^{2}\right)$ :

$$
\begin{aligned}
\mathrm{XT}_{4}^{1}\left(u_{l} \| v_{l}\right) & =p_{1}\left(p_{1}^{-1}\left(u_{l}\right) \oplus p_{1}^{-1}\left(v_{l}\right)\right), \\
\mathrm{XT}_{4}^{2}\left(u_{h} \| v_{h}\right) & =p_{2}\left(p_{2}^{-1}\left(u_{h}\right) \oplus p_{2}^{-1}\left(v_{h}\right)\right) .
\end{aligned}
$$

Tables $\mathrm{XT}_{4}^{1}$ and $\mathrm{XT}_{4}^{2}$ are calculated at the same time as $P$, and stored in memory. An 8-bit XOR function, denoted by $\mathrm{XT}_{8}$, is then computed using those table look-ups (for $u, v \in \mathbb{F}_{2}^{8}$ ):

$$
\mathrm{XT}_{8}(u, v)=\mathrm{XT}_{4}^{2}\left(u_{h} \| v_{h}\right) \| \mathrm{XT}_{4}^{1}\left(u_{l} \| v_{l}\right) .
$$

- Randomised MixColumns is computed as a combination of doubling and XOR operations. To calculate randomised MixColumns from the $P\left(a_{i}\right)$ 's (the $P$ representations of the bytes of the AES state), the XOR operations are computed using the $\mathrm{XT}_{8}$ function in Eq. (8) . For the doubling operations, Coron defined a function $D_{2}$, such that when applied to $u=P(x)$, we get $D_{2}(P(x))=P(\{02\} \bullet x)$ (where $\{\cdot\}$ denotes hexadecimal notation, and • denotes multiplication modulo $\left.x^{8}+x^{4}+x^{3}+x+1\right)$. The $P$-representation of the first byte of the MixColumns output is then calculated using:

$$
P\left(a_{0}^{n e w}\right)=\operatorname{XT}_{8}\left(D_{2}\left(a_{0}^{\prime}\right), \operatorname{XT}_{8}\left(D_{2}\left(a_{1}^{\prime}\right), \operatorname{XT}_{8}\left(a_{1}^{\prime}, \mathrm{XT}_{8}\left(a_{2}^{\prime}, a_{3}^{\prime}\right)\right)\right)\right),
$$

where $a_{i}^{\prime}$ denotes the $P$-representation of $a_{i}$. The other bytes in the randomised MixColumns output can be similarly calculated.

At the completion of the last encryption round, the inverse permutation $P^{-1}$ is applied to each byte of the AES state, revealing the ciphertext.

\section{Security of Randomized AES against First-Order SCA}

\subsection{Examining the Proof of Security}

In [5], the author proposes the following Lemma to argue that the randomised AES implementation is resistant against first-order SCA:

Lemma 1. For a fixed key and input message, every intermediate byte that is computed in the course of the randomised AES algorithm has the uniform distribution in $\{0,1\}^{8}$. 
In [5], the proof of Lemma 1 is based on the fact that any intermediate AES data $W$ is assumed to be represented as $P(W)=P_{2}\left(W_{h}\right) \| P_{1}\left(W_{l}\right)$. However, this assumption is incorrect for the implementation described in [5] and recalled in Section 3.2. Indeed, when $\mathrm{XT}_{8}(P(X), P(Y))$ is computed (Eq. (8)), the two functions $\mathrm{XT}_{4}^{1}$ and $\mathrm{XT}_{4}^{2}$ are parameterized with the intermediate variables $P_{1}\left(X_{l}\right) \| P_{1}\left(Y_{l}\right)$ and $P_{2}\left(X_{h}\right) \| P_{2}\left(Y_{h}\right)$ respectively. Namely, the same permutation $P_{1}$ (resp. $P_{2}$ ) is applied to the lowest and the highest nibbles of the intermediate data $W=X_{l} \| Y_{l}$ (resp. $W=X_{h} \| Y_{h}$ ). In this case $W$ is not of the form $P(W)$; therefore, the statement made in $[5$ to prove Lemma1 1 is incorrect. Actually, not only the proof but the Lemma itself is incorrect. If two nibbles are equal, e.g. $X_{l}=Y_{l}$, then their $P_{1}$-representations will also be equal, i.e. $P_{1}\left(X_{l}\right)=P_{1}\left(Y_{l}\right)$, irrespective of $P_{1}$. Otherwise, if $X_{l} \neq Y_{l}$, then $P_{1}\left(X_{l}\right)$ and $P_{1}\left(Y_{l}\right)$ behave like two independent random variables, except that they cannot be equal. This implies that the variable $P_{1}\left(X_{l}\right) \| P_{1}\left(Y_{l}\right)$ will have two different non-uniform distributions depending on whether $X_{l}$ equals $Y_{l}$ or not. This gives rise to first-order leakage.

\subsection{First-Order Leakage Points}

In the randomised AES, the function $\mathrm{XT}_{8}$ is employed to securely implement every bitwise addition between 8-bit words. To compute the $P$-representation of $X \oplus Y$ from the $P$-representations $X^{\prime}=P(X)$ and $Y^{\prime}=P(Y)$, the following successive operations are processed:

$$
\begin{aligned}
& \text { 1. } R_{1} \leftarrow \mathrm{XT}_{4}^{1}\left(X_{l}^{\prime} \| Y_{l}^{\prime}\right) \\
& \text { 2. } R_{2} \leftarrow \mathrm{XT}_{4}^{2}\left(X_{h}^{\prime} \| Y_{h}^{\prime}\right) \\
& \text { 3. output } \leftarrow \quad R_{2} \| R_{1}
\end{aligned}
$$

Register output contains $P(X \oplus Y)$ at the end of the processing above. Let us focus on the intermediate result $R_{1}$ (the same analysis also holds for $R_{2}$ ). It is computed by accessing the table $\mathrm{XT}_{4}^{1}$ at address $Z=X_{l}^{\prime} \| Y_{l}^{\prime}$ which, by construction, satisfies:

$$
Z=P_{1}\left(X_{l}\right) \| P_{1}\left(Y_{l}\right) .
$$

As discussed in Section 4.1 the manipulation of $Z$ therefore induces a first-order leakage in the AES implementation, whenever $\left(X_{l}, Y_{l}\right)$ statistically depends on a secret information and a known data. This condition is satisfied when $\mathrm{XT}_{8}$ is used to process the randomised AddRoundKey and randomised MixColumns operations during the first round of AES:

- [Randomised AddRoundKey] During this step, $\mathrm{XT}_{8}$ takes the pair $(P(A), P(K))$ as operand, where $K$ is a round key byte and $A$ is a known byte of the AES state. In this case, (10) becomes:

$$
Z=P_{1}\left(A_{l}\right) \| P_{1}\left(K_{l}\right) .
$$

- [Randomised MixColumns] During this step, $\mathrm{XT}_{8}$ takes the pair $\left(A_{1}^{\prime}, A_{2}^{\prime}\right)=$ $\left(P\left(S\left[A_{1} \oplus K_{1}\right]\right), P\left(S\left[A_{2} \oplus K_{2}\right]\right)\right)$ as operand, with $S$ denoting the AES S-box, 
with $A_{1}$ and $A_{2}$ being two known bytes of the AES state and with $K_{1}$ and $K_{2}$ being two round-key bytes.

In this case, (10) becomes:

$$
Z=P_{1}\left(S\left[A_{1} \oplus K_{1}\right]_{l}\right) \| P_{1}\left(S\left[A_{2} \oplus K_{2}\right]_{l}\right),
$$

where $S[\cdot]_{l}$ denotes the lowest nibble of $S[\cdot]$.

Both of the leakage points described above are first-order flaws, since they depend on a single intermediate variable $Z$. In the next sections, we will develop firstorder side-channel attacks, that exploit these first order leakages. In both attacks, we will use the notation $Z\left(k_{l}\right)$ (resp. $Z\left(k_{1}, k_{2}\right)$ ) for the random variable $Z \mid\left(K_{l}=\right.$ $\left.k_{l}\right)$ (resp. $Z \mid\left(K_{1}=k_{1}, K_{2}=k_{2}\right)$ ), each time we need to specify which $\operatorname{key}(\mathrm{s}) Z$ is related to. The random variable corresponding to the leakage on $Z$ shall be denoted by $L$. They are related through the following relationship:

$$
L=\varphi(Z)+B
$$

where $\varphi$ denotes a deterministic function called the leakage function and $B$ denotes independent noise. We shall use the notation $L\left(k_{l}\right)$ (resp. $L\left(k_{1}, k_{2}\right)$ ) when we need to specify the key(s) involved in the leakage measurements.

\section{Attacking the Randomised AddRoundKey Operation}

There are currently two main ways to perform an attack on the manipulation of a random variable $Z$. The first method relies on affine statistical dependencies (for example CPA), whereas the second method relies on any kind of statistical dependency (for example MIA). Here, we describe a CPA attack on the first use of randomised AddRoundKey (performing an MIA attack on randomised AddRoundKey is less pertinent, as will be discussed in Section [6).

\subsection{CPA Preliminaries}

In a CPA 3, the attacker must know a good affine approximation $\hat{\varphi}$ of $\varphi$. It is common to choose the Hamming Weight (HW) function for $\hat{\varphi}$, as this is known to be a good leakage model for devices such as 8-bit microcontrollers. The attacker must also know a good affine approximation $\hat{Z}$ of $Z$. Based on these assumptions, key candidates $k_{l}^{\star}$ are discriminated by testing the correlation between $\hat{\varphi}\left(\hat{Z}\left(k_{l}^{\star}\right)\right)$ and $L\left(k_{l}\right)$, for a sample of leakage measurements from the target device, and the corresponding known plaintexts.

Here, our attack targets the use of $\mathrm{XT}_{8}$ when the first randomised AddRoundKey operation is performed. We assume that a sample of $N$ leakages $\left(\ell_{i}\right)$ has been measured for $N$ known lowest nibbles $\left(a_{i}\right)$ of the AES state. Due to (11) and (13), the $\ell_{i}$ 's and the $a_{i}$ 's satisfy the following relation:

$$
\ell_{i}=\varphi\left(p_{1, i}\left(a_{i}\right) \| p_{1, i}\left(k_{l}\right)\right)+b_{i},
$$


for $1 \leq i \leq N$, where $b_{i}$ denotes the value of the noise for the $i$ th leakage measurement and where $p_{1, i}$ denotes the permutation used at the time of the $i$ th measurement.

To test a hypothesis $k_{l}^{\star}$ on $k_{l}$, the following Pearson's coefficient $\widehat{\rho}_{k_{l}^{\star}}$ is computed for an appropriate prediction function $f$ :

$$
\widehat{\rho}_{k_{l}^{\star}}=\widehat{\rho}\left(\left(\ell_{i}\right)_{i},\left(f\left(a_{i}, k_{l}^{\star}\right)\right)_{i}\right) .
$$

If $f$ has been well chosen, the expected key will satisfy $k_{l}=\operatorname{argmax}_{k_{l}^{\star}}\left|\widehat{\rho}_{k_{l}^{\star}}\right|$. This is the case for leakage functions $\varphi$ in (14) where $\mathrm{E}\left[\varphi\left[Z\left(k_{l}\right)\right]\right]$ is not constant on $\mathcal{K}_{l}$ (recall that $Z\left(k_{l}\right)$ equals $P_{1}(A) \| P_{1}\left(k_{l}\right)$ ). Almost all functions $\varphi$ satisfy this condition. However, this is not the case for functions $\varphi$ where $\varphi(X \| Y)=$ $\varphi(X)+\varphi(Y)($ e.g. $\varphi=\mathrm{HW})$. For those leakage functions, Pearson's coefficient (15) is not a sound key-distinguisher when applied directly to the leakages $\ell_{i}$ 's. Indeed, in this case, (3) and $\sigma\left[\varphi\left[Z\left(K_{l}\right)\right] \mid K_{l}\right]=0$ imply that $\rho\left[L\left(k_{l}\right), f\left(A, k_{l}\right)\right]$ is null, regardless of the prediction function $f$. For such functions $\varphi$, it makes sense (see for instance [18]) to focus on higher order moments, and to compute the following Pearson's coefficient for an appropriate function $f$, which may differ from the case when $o=1$ :

$$
\widehat{\rho}_{k_{l}^{\star}}=\widehat{\rho}\left(\left(\left(\ell_{i}-\bar{\ell}\right)^{o}\right)_{i},\left(f\left(a_{i}, k_{l}^{\star}\right)\right)_{i}\right)
$$

For instance, if $\varphi=\mathrm{HW}$, then the second order centered moments of the $\varphi\left[Z\left(k_{l}\right)\right]$ 's are different, so (16) must be computed for $o=2$.

Remark 2. For $o=1$ (i.e. when the CPA focuses on the means), there is no need to center the leakage measurements and the term $\bar{\ell}$ can be omitted. In the other cases, centering the leakage measurements (and thus the predictions) improves the CPA efficiency (see [17]).

When a good approximation $\hat{\varphi}$ of $\varphi$ is assumed to be known, the efficiency of the CPA relies on the prediction function $f$ that is chosen. This is especially true in our case where data is not simply masked by the addition of a random value, but by a random permutation, so that removing the effect of the masking (even biased) is difficult. Designing a prediction function $f$, such that a CPA involving this function in (16) succeeds, is not straightforward. Therefore, to exploit the flaw in (11) using a CPA attack, we need to exhibit a sound prediction function $f$.

\subsection{Designing $f_{\text {opt }}$}

The target intermediate variable $Z$ in (11) takes the general form $P_{1}(X) \| P_{1}(Y)$, where $P_{1}, X$ and $Y$ are random variables and where $Y$ depends on $k_{l}$. In [17], Prouff et al. showed that for every function $\mathcal{C}: L \mapsto \mathcal{C}(L)$, the optimal prediction function $f_{\text {opt }}$ is the function $x, y \mapsto \mathrm{E}\left[\mathcal{C}\left(L\left(k_{l}\right)\right) \mid X=x, Y=y\right]$. In our case, $\mathcal{C}\left(L\left(k_{l}\right)\right)$ equals $\left(L\left(k_{l}\right)-\mathrm{E}\left[L\left(k_{l}\right)\right]\right)^{o}$ for a given order $o$. To mount the attack, we need an analytical expression for the function $f_{\text {opt }}$ so that it can be estimated even when no 
information on the noise parameters is known (non-profiling attacks). Therefore, we conducted an analytical study of the function $f_{\text {opt }}$ defined by:

$$
f_{\text {opt }}(x, y)=\mathrm{E}\left[\left(L\left(k_{l}\right)-\mathrm{E}\left[L\left(k_{l}\right)\right]\right)^{o} \mid X=x, Y=y\right],
$$

for $o \in \mathbb{N}$, for $L$ equal to $\hat{\varphi}(Z)+B$, for $B \sim \mathcal{N}\left(\varepsilon, \sigma^{2}\right)$ and for $Z$ equal to $P_{1}(X) \| P_{1}(Y)$ with $X, Y \sim \mathcal{U}\left(\mathbb{F}_{2}^{n}\right)$ and $P_{1}$ is a random variable over $\mathcal{P}$.

Below we state the results of our analysis (the derivations of the formulas are given in Appendix $(\mathrm{A})$ :

- To compute (16), we suggest using the prediction function $f$ defined for every $\left(a_{i}, k_{l}^{\star}\right) \in\left(\mathbb{F}_{2}^{4}\right)^{2}$ by:

$$
f\left(a_{i}, k_{l}^{\star}\right)=\sum_{p_{1} \in \mathcal{P}}\left(\hat{\varphi}\left(p_{1}\left(a_{i}\right) \| p_{1}\left(k_{l}^{\star}\right)\right)-\mathrm{E}\left[\hat{\varphi}_{k_{l}^{\star}}\right]\right)^{o} \mathrm{p}\left[P_{1}=p_{1}\right],
$$

where:

$$
\mathrm{E}\left[\hat{\varphi}_{k_{l}^{\star}}\right]=2^{-4} \sum_{a \in \mathbb{F}_{2}^{4}} \sum_{p_{1} \in \mathcal{P}} \hat{\varphi}\left(p_{1}(a) \| p_{1}\left(k_{l}^{\star}\right)\right) \mathrm{p}\left[P_{1}=p_{1}\right] .
$$

For $o \in\{1,2\}$, it is argued in Appendix $\mathrm{A}$ that the functions $f$ above are affine equivalent to $f_{\text {opt }}$.

- Let $\delta_{x}(y)$ be the function defined by $\delta_{x}(y)=1$ if $x=y$, and $\delta_{x}(y)=0$ otherwise. If we assume that $o=2, \hat{\varphi}=\mathrm{HW}$, and that $P_{1}$ has a uniform distribution over $\mathcal{P}$, we suggest using the following function:

$$
f_{\text {opt }}\left(a_{i}, k_{l}^{\star}\right)=\delta_{a_{i}}\left(k_{l}^{\star}\right),
$$

which is affine equivalent to $f_{o p t}$.

\subsection{Attack Results}

In the attack simulations presented in Table 1, we give an estimation of the minimum number of measurements required to achieve the success rate 0.9 for $P_{1} \sim \mathcal{U}(\mathcal{P})$ (where $\mathcal{P}$ is designed as proposed in [5]) and $\varphi=\hat{\varphi}=$ HW. In this case, Pearson coefficients have been computed between $\left(\left(\ell_{i}-\bar{\ell}\right)^{2}\right)_{i}$ and $\left(f_{\text {opt }}\left(a_{i}, k_{l}^{\star}\right)\right)_{i}$ for the function $f_{\text {opt }}$ defined in (18) for $o=2$. This success rate is defined as the ratio of successful attacks involving $N$ measurements to the number of attacks involving $N$ measurements. We assumed that an attack is successful if the highest correlation is attained for the correct key. The simulations show that for noiseless measurements, key nibbles can be successfully recovered from the randomised AddRoundKey operation using only 100 power traces. We also carried out the CPA attack on a practical smart card implementation of the randomised AES, as described by [5. We used a Silvercard, which contains a programmable 8-bit PIC16F877 microprocessor, and verified that the power consumption of the card leaks information in the HW model. For each plaintext sent to the card, the encryption operation was performed ten 
Table 1. Num. of measurements required in simulated CPA attack on randomised AddRoundKey

\begin{tabular}{|c|c|}
\hline $\begin{array}{c}\text { Noise standard } \\
\text { deviation }\end{array}$ & $\begin{array}{c}\text { Number of } \\
\text { measurements }\end{array}$ \\
\hline 0 & 100 \\
0.5 & 1,000 \\
1 & 1,500 \\
2 & 4,500 \\
5 & 60,000 \\
7 & 230,000 \\
10 & 900,000 \\
\hline
\end{tabular}

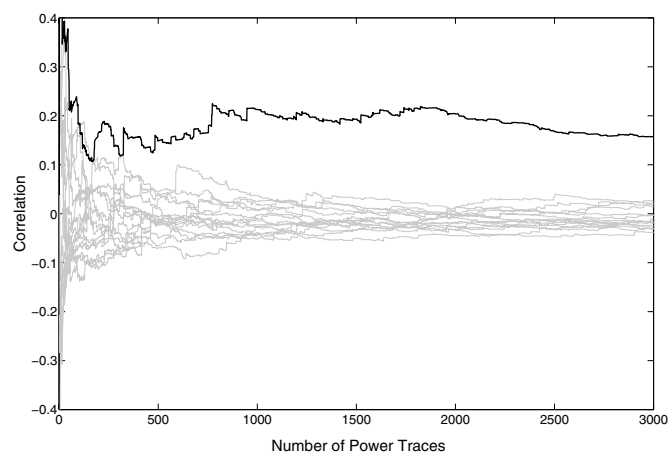

Fig. 1. CPA attack on smart card implementation of randomised AddRoundKey

times (with the same random values used to generate the permutation tables) and an average trace of the power consumption was recorded, in order to reduce the effects of acquisition noise. For the attack, we calculated the correlations between $\left(\left(\ell_{i}-\bar{\ell}\right)^{2}\right)_{i}$ and $\left(f_{\text {opt }}\left(a_{i}, k_{l}^{\star}\right)\right)_{i}$ for the simplified function $f_{\text {opt }}$ defined in (20). The results of the attack are shown in Fig. 1 for various numbers of power traces. The correlation for the correct key nibble is highlighted, showing that the correct key nibble can be recovered using fewer than 1,000 plaintext/power trace pairs.

\section{Attacking the Randomised MixColumns Operation}

In this section, we describe CPA and MIA attacks that target the use of $\mathrm{XT}_{8}$ when the first MixColumns operation is performed. These attacks are of interest, because they allow recovery of two key bytes ( $c f$. Eq. (12)), as opposed to a single key nibble when the AddRoundKey operation is targeted. We assume that a sample of $N$ leakages $\left(\ell_{i}\right)_{i}$ has been measured for $N$ pairs of known AES state values $\left(\left(a_{1, i}, a_{2, i}\right)\right)_{i}$ (where $a_{j, i}$ denotes the known value of byte $a_{j}$ at the time of the $i$ th measurement $\ell_{i}$ ). Due to (12) and (13), the $\ell_{i}$ 's and the $a_{j, i}$ 's satisfy the following relation:

$$
\ell_{i}=\varphi\left(p_{1, i}\left(S\left[a_{1, i} \oplus k_{1}\right]_{l}\right) \| p_{1, i}\left(S\left[a_{2, i} \oplus k_{2}\right]_{l}\right)+b_{i},\right.
$$

where $b_{i}$ and $p_{1, i}$ are as defined for Eq. (14).

\subsection{MIA Preliminaries}

In MIA attacks [8], key candidates $k^{\star}$ are discriminated by estimating the mutual information $\mathrm{I}\left(\hat{\varphi}\left(\hat{Z}\left(k^{\star}\right)\right) ; L(k)\right)$. In an MIA, the attacker is potentially allowed to make weaker assumptions on $\varphi$ and on $Z$ than in the CPA. Indeed, rather than a good affine approximation of $\varphi$ and of $Z$, we only require a pair $(\hat{\varphi}, \hat{Z})$ such that 
$\mathrm{I}(\hat{\varphi}(\hat{Z}(k)) ; L(k))$ is non-negligible when the good key $k$ is tested (which may happen even if $\rho(\hat{\varphi}(\hat{Z}(k)), L(k))=0$ ) (see 116] for more details). Therefore, we do not require a lengthy derivation for a prediction function $f_{\text {opt }}$, as was required in Section 5.2 for the CPA. After assuming that a good approximation $\hat{\varphi}$ of the leakage function $\varphi$ is known, an MIA attack can be performed by estimating the mutual information between the random variable $L$ associated with the leakage measurements $\ell_{i}$ in (21) and the prediction function $\hat{\varphi}\left(S\left[A_{1} \oplus\right.\right.$ $\left.\left.k_{1}^{\star}\right]_{l} \| S\left[A_{2} \oplus k_{2}^{\star}\right]_{l}\right)$, for various hypotheses $\left(k_{1}^{\star}, k_{2}^{\star}\right)$ on key bytes $\left(k_{1}, k_{2}\right)$. The mutual information will attain its maximum value for the correct set of key hypotheses.

Remark 3. As noted in Sec. 5, it was less pertinent to use mutual information as a distinguisher when attacking the randomised AddRoundKey operation. The main reason for this is that when $\varphi$ is the Hamming weight function, the conditional random variable $\varphi(Z(k))$ has the same entropy for each $k$. As discussed in 816 , a way to deal with this issue is to focus on the mutual information between $\varphi(Z(k))$ and predictions in the form $\hat{\varphi} \circ \psi\left(\hat{Z}\left(k^{\star}\right)\right)$, where $\psi$ is any non-injective function. Even if this approach enables recovery of the key, we checked that for various functions $\psi$ (in particular for functions $\psi$ selecting less than 8 bits in $\hat{Z}(k))$, MIA attacks were much less efficient than CPA.

\subsection{Attack Results}

In simulation, we tested both an MIA attack and a CPA attack, targeting the first call to $\mathrm{XT}_{8}$ in the first MixColumns operation. For the MIA, we used the Kernel Density and Parametric estimation methods described in [16] to estimate the mutual information. For the same reasons as given in Sec.5.2 (and Appendix A), the CPA simulations used the pre-processing described in Eq. (16), and the following prediction function:

$$
f_{\text {opt }}\left(a_{1, i}, k_{1}^{\star}, a_{2, i}, k_{2}^{\star}\right)=\delta_{\left(S\left[a_{1, i} \oplus k_{1}^{\star}\right]_{l}\right)}\left(S\left[a_{2, i} \oplus k_{2}^{\star}\right]_{l}\right)
$$

In the attack simulations presented in Table 2, we give a rough estimation of the minimum number of measurements required to achieve the success rate 0.9 for the different distinguishers. In these experiments, one key byte was fixed at the correct value, and the distinguishers were calculated for the $2^{8}$ values of the second key byte. Fewer measurements are required for a successful

Table 2. Num. measurements required in MIA and CPA attacks on randomised MixColumns (where '-' implies no successful result with up to 1 million measurements)

\begin{tabular}{|c|c|c|c|c|c|c|c|c|c|}
\hline Noise standard deviation & 0 & 0.5 & 1 & 2 & 5 & 7 & 10 & 15 & 20 \\
\hline Nb of measurements [MIA with Kernel] & 2,500 & 20,000 & 60,000 & 290,000 & - & - & - & - & - \\
\hline Nb of measurements [Parametric MIA] & na & 3,000 & 4,000 & 25,000 & 250,000 & 500,000 & 800,000 & - & - \\
\hline \hline Nb of measurements [CPA with $f_{\text {opt }}$ ] & 1,000 & 1,000 & 1,500 & 6,500 & 120,000 & 550,000 & - & - & - \\
\hline
\end{tabular}




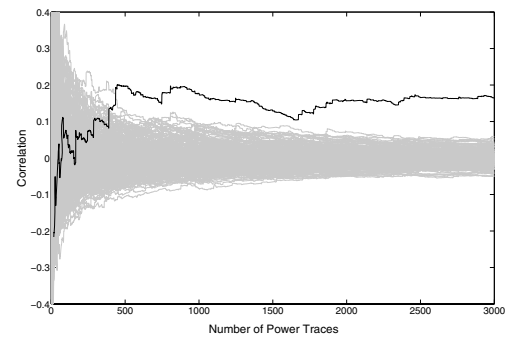

Fig. 2. CPA attack on smart card implementation of randomised MixColumns

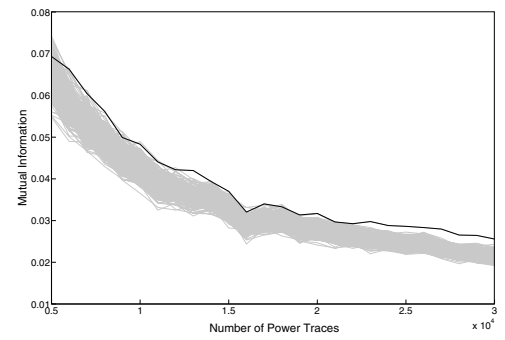

Fig. 3. MIA attack on smart card implementation of randomised MixColumns

attack using CPA than are required when using MIA, for low-noise measurements. This is to be expected, since in the simulations, the HW of the attack variable leaks perfectly, so there is a linear relation between the deterministic part of the leakage and the prediction. MIA is more useful when the relationship between the leakage and the prediction is non-linear. It is interesting to note that when the measurements are noisy, the parametric MIA attack is more efficient than the CPA attack (even in this simulation context that is favourable to CPA).

These attacks were also verified using measurements from the smart card implementation, as shown in Figures 2 and 3 (where the distinguisher for the correct key byte is highlighted). Since the noise in these acquisitions has been reduced due to averaging, the CPA succeeds in fewer measurements $(\sim 2,000$ power traces) than an MIA attack ( 23, 000 traces, using the histogram method to estimate the mutual information $[8]$ ).

\section{Conclusion}

In this paper, we have shown that first-order flaws exist in the permutation tables countermeasure proposed in [5]. In order to exploit this leakage, two attacks have been developed. The first attack applies the recent work of [17 to develop an optimal prediction function for use in a correlation-based attack. The second attack is based on mutual information analysis, and uses estimation methods proposed by [16. The new attacks were verified in both simulation and practice. In the extended version of this paper [15], we suggest a patch for the permutation tables countermeasure, thereby removing the first-order leakage. It is interesting to note that even if the permutation tables countermeasure is flawed, exploiting this flaw requires more traces than, for instance, an attack on a flawed masking scheme. Therefore, an avenue for further research is to examine the HO-SCA resistance of the (patched) permutation tables countermeasure, as it may also be more HO-SCA resistant than masking. 


\section{References}

1. Aumonier, S.: Generalized Correlation Power Analysis. Published in the Proceedings of the Ecrypt Workshop Tools For Cryptanalysis 2007(2007)

2. Blömer, J., Merchan, J.G., Krummel, V.: Provably Secure Masking of AES. In: Handschuh, H., Hasan, M.A. (eds.) SAC 2004. LNCS, vol. 3357, pp. 69-83. Springer, Heidelberg (2004)

3. Brier, É., Clavier, C., Olivier, F.: Correlation Power Analysis with a Leakage Model. In: Joye, M., Quisquater, J.-J. (eds.) CHES 2004. LNCS, vol. 3156, pp. 16-29. Springer, Heidelberg (2004)

4. Chari, S., Jutla, C.S., Rao, J.R., Rohatgi, P.: Towards Sound Approaches to Counteract Power-Analysis Attacks. In: Wiener, M. (ed.) CRYPTO 1999. LNCS, vol. 1666, pp. 398-412. Springer, Heidelberg (1999)

5. Coron, J.-S.: A New DPA Countermeasure Based on Permutation Tables. In: Ostrovsky, R., De Prisco, R., Visconti, I. (eds.) SCN 2008. LNCS, vol. 5229, pp. 278-292. Springer, Heidelberg (2008)

6. Coron, J.-S., Giraud, C., Prouff, E., Rivain, M.: Attack and Improvement of a Secure S-Box Calculation Based on the Fourier Transform. In: Oswald, E., Rohatgi, P. (eds.) CHES 2008. LNCS, vol. 5154, pp. 1-14. Springer, Heidelberg (2008)

7. Fumaroli, G., Mayer, E., Dubois, R.: First-Order Differential Power Analysis on the Duplication method. In: Srinathan, K., Rangan, C.P., Yung, M. (eds.) INDOCRYPT 2007. LNCS, vol. 4859, pp. 210-223. Springer, Heidelberg (2007)

8. Gierlichs, B., Batina, L., Tuyls, P., Preneel, B.: Mutual information analysis. In: Oswald, E., Rohatgi, P. (eds.) CHES 2008. LNCS, vol. 5154, pp. 426-442. Springer, Heidelberg (2008)

9. Golić, J., Tymen, C.: Multiplicative Masking and Power Analysis of AES. In: Kaliski Jr., B.S., Koç, Ç.K., Paar, C. (eds.) CHES 2002. LNCS, vol. 2523, pp. 198-212. Springer, Heidelberg (2003)

10. Joye, M., Paillier, P., Schoenmakers, B.: On Second-order Differential Power Analysis. In: Rao, J.R., Sunar, B. (eds.) CHES 2005. LNCS, vol. 3659, pp. 293-308. Springer, Heidelberg (2005)

11. Kocher, P.: Timing attacks on implementations of Diffie-Hellman, RSA, DSS and other systems. In: Koblitz, N. (ed.) CRYPTO 1996. LNCS, vol. 1109, pp. 104-113. Springer, Heidelberg (1996)

12. Menezes, A.J., van Oorschot, P.C., Vanstone, S.A.: Handbook of Applied Cryptography. CRC Press, Boca Raton (1997)

13. Messerges, T.S.: Using Second-order Power Analysis to Attack DPA Resistant software. In: Paar, C., Koç, Ç.K. (eds.) CHES 2000. LNCS, vol. 1965, pp. 238-251. Springer, Heidelberg (2000)

14. Piret, G., Standaert, F.-X.: Security Analysis of Higher-Order Boolean Masking Schemes for Block Ciphers (with Conditions of Perfect Masking). IET Information Security 2(1), March 1-11 (2008)

15. Prouff, E., McEvoy, R.: First-Order Side-Channel Attacks on the Permutation Tables Countermeasure - Extended Version. To appear on the Cryptology ePrint Archive (2009), http://eprint.iacr.org

16. Prouff, E., Rivain, M.: Theoretical and Practical Aspects of Mutual Information Based Side Channel Analysis. In: Abdalla, M., et al. (eds.) ACNS 2009. LNCS, vol. 5536, pp. 502-521. Springer, Heidelberg (2009) 
17. Prouff, E., Rivain, M., Bévan, R.: Statistical Analysis of Second Order Differential Power Analysis. IEEE Transactions on Computers 58(6), 799-811 (2009)

18. Soong, T.T.: Fundamentals of Probability and Statistics for Engineers, 3rd edn. John Wiley \& Sons, Ltd, Chichester (2004)

19. Waddle, J., Wagner, D.: Toward Efficient Second-order Power Analysis. In: Joye, M., Quisquater, J.-J. (eds.) CHES 2004. LNCS, vol. 3156, pp. 1-15. Springer, Heidelberg (2004)

\section{A Derivation of $f_{\text {opt }}$ for CPA Attacks}

This section aims at deriving analytical expressions for the function $f_{\text {opt }}$. We begin with the expression $f_{\text {opt }}(x, y)=\mathrm{E}\left[(L-\mathrm{E}[L])^{\circ} \mid X=x, Y=y\right]$ (Eq. (17)), where for clarity reasons and because there is no ambiguity, we use the notation $L$ in place of $L\left(k_{l}\right)$. We recall that the random variable $L$ is assumed to satisfy $L=\hat{\varphi}(Z)+B$ and that $Z$ equals $P_{1}(X) \| P_{1}(Y)$ with $P_{1} \sim \mathcal{U}(\mathcal{P})$. Since the expectation is linear and the random variables $B$ and $(X, Y)$ are independent, developing $(L-\mathrm{E}[L])^{\circ}$ leads to:

$$
\begin{aligned}
f_{o p t}(x, y)=\mathrm{E}\left[(\hat{\varphi}(Z)-m)^{o} \mid(X, Y)=(x, y)\right]+\mu_{o} \\
+\sum_{i=1}^{o-1}\left(\begin{array}{l}
o \\
i
\end{array}\right) \mu_{o-i} \mathrm{E}\left[(\hat{\varphi}(Z)-m)^{i} \mid(X, Y)=(x, y)\right],
\end{aligned}
$$

where $m$ denotes the mean $\mathrm{E}[\hat{\varphi}(Z)]$ and $\mu_{i}$ denotes the $i$ th order central moment of $B \sim \mathcal{N}\left(\varepsilon, \sigma^{2}\right)$. Let us notice that since $\mu_{1}$ is zero, the sum in (23) can start from $i=2$.

Example 1. For o equal to 1 and 2, we respectively have:

$$
f_{\text {opt }}(x, y)=\mathrm{E}[\hat{\varphi}(Z)-m \mid(X, Y)=(x, y)]
$$

and

$$
f_{\text {opt }}(x, y)=\mathrm{E}\left[(\hat{\varphi}(Z)-m)^{2} \mid(X, Y)=(x, y)\right]+\mu_{2} .
$$

The prediction function given in (18) corresponds to the development of the terms in (23) that do not depend on noise parameters. It must be noticed that in the cases $o=1$ and $o=2$, such an estimation of $f_{o p t}$ is perfect since the terms that depend on noise parameters are either null or constant.

Henceforth, we assume that $\mathcal{P}$ is the set of all permutations over $\mathbb{F}_{2}^{n}$ and that $P_{1}$ is a random variable with uniform distribution over $\mathcal{P}$. This assumption is very favorable to the permutation table countermeasure since it implies that the choice of the masking permutation $P_{1}$ is not reduced to a sub-class of the set of permutations over $\mathbb{F}_{2}^{n}$.

We now focus on the non-noisy term in (23), namely on the mean $\mathrm{E}\left[(\hat{\varphi}(Z)-m)^{o} \mid(X, Y)=(x, y)\right]$. Moreover, we denote this conditional mean 
by $g(x, y)$, and define $\delta_{x}(y)$ s.t. $\delta_{x}(y)=1$ if $y=x$ and $\delta_{x}(y)=0$ otherwise (resp. $\left.\overline{\delta_{x}}(y)=1-\delta_{x}(y)\right)$. We have the following Lemma:

Lemma 2. Let $X$ and $Y$ be two random variables with uniform distributions over $\mathbb{F}_{2}^{n}$ and let $P_{1}$ be a random variable uniformly distributed over the set of all permutations over $\mathbb{F}_{2}^{n}$. Then for every $\hat{\varphi}$ the function $g$ is 2 -valued and satisfies:

$$
g(x, y)=\frac{2^{n} \delta_{x}(y)-1}{2^{n}-1} E\left[(\hat{\varphi}(I|| I)-m)^{o}\right]+\frac{2^{n} \overline{\delta_{x}}(y)}{2^{n}-1} E\left[(\hat{\varphi}(I|| J)-m)^{o}\right],
$$

where $I$ and $J$ are two independent random variables with uniform distribution over $\mathbb{F}_{2}^{n}$.

Proof. For every $(x, y) \in \mathbb{F}_{2}^{2 n}$ we have

$$
g(x, y)=\sum_{i, j \in \mathbb{F}_{2}^{n}}(\hat{\varphi}(i \| j)-m)^{o} \mathrm{p}\left[P_{1}(x)=i, P_{1}(y)=j\right]
$$

Since $P_{1}$ is assumed to have uniform distribution over the set of permutations over $\mathbb{F}_{2}^{n}$, for every $(x, y) \in\left(\mathbb{F}_{2}^{n}\right)^{2}$ s.t. $x \neq y$ we have:

$$
\mathrm{p}\left[P_{1}(x)=i, P_{1}(y)=j\right]=\left\{\begin{array}{cc}
1 / 2^{n}\left(2^{n}-1\right) & \text { if } i \neq j \\
0 & \text { otherwise. }
\end{array}\right.
$$

If $x=y$, we have

$$
\mathrm{p}\left[P_{1}(x)=i, P_{1}(y)=j\right]=\left\{\begin{array}{cc}
1 / 2^{n} & \text { if } i=j \\
0 & \text { otherwise. }
\end{array}\right.
$$

Combining (25) and (26) gives (24).

When the estimation $\hat{\varphi}$ is the Hamming weight over $\mathbb{F}_{2}^{2 n}$, (24) can be further developed. Indeed, in this case we have:

$$
g(x, y)=\frac{2^{n} \delta_{x}(y)-1}{2^{n}-1} 2^{o} \mathrm{E}\left[\left(\operatorname{HW}(I)-\frac{n}{2}\right)^{o}\right]+\frac{2^{n} \overline{\delta_{x}}(y)}{2^{n}-1} \mathrm{E}\left[(\operatorname{HW}(I \| J)-n)^{o}\right],
$$

since $m$ equals $\mathrm{E}[\mathrm{HW}]$, i.e. $n$ when $\mathrm{HW}$ is defined over $\mathbb{F}_{2}^{2 n}$.

As $\mathrm{E}[\mathrm{HW}(I)]$ equals $\frac{n}{2}$ and $\mathrm{E}[\mathrm{HW}(I \| J)]$ equals $n$, the function $g$ is constant equal to 0 when $o=1$. For $o=2$, it satisfies:

$$
g(x, y)=\delta_{x}(y) \frac{n 2^{n-1}}{2^{n}-1}+\frac{n\left(2^{n-1}-1\right)}{2^{n}-1}
$$

since we have $\mathrm{E}\left[\left(\mathrm{HW}(I)-\frac{n}{2}\right)^{2}\right] \quad$ (resp. $\left.\mathrm{E}\left[(\mathrm{HW}(I \| J)-n)^{2}\right]\right)$ equal to $\operatorname{Var}[\mathrm{HW}(I)]=\frac{n}{4}\left(\operatorname{resp} . \operatorname{Var}[\mathrm{HW}(I \| J)]=\frac{n}{2}\right)$. 
For $o=2$, (27) implies that $f_{\text {opt }}$ is an affine increasing function of $\delta_{x}(y)$. Since the correlation coefficient is invariant for any affine transformation of one or both of its parameters, the function $x, y \mapsto \delta_{x}(y)$ itself (and every affine transformation of it) is actually an optimal prediction function for $o=2$. Hence, in its simplest form the optimal function for $o=2$ is defined for every $(x, y) \in \mathbb{F}_{2}^{n 2}$ as:

$$
f_{\text {opt }}(x, y)=\delta_{x}(y)
$$

For $o=2$, the function $f_{\text {opt }}$ in (28) can be applied to conduct CPA attacks in the particular case of Coron's construction of $\mathcal{P}$ (which is not the set of all permutations over $\{0, \ldots, 15\}$ but a subset of it with cardinality $16^{4}$ ), without losing a significant factor in attack efficiency (in terms of number of leakage measurements). 